\title{
BIMODALITY AND LITHIUM ABUNDANCE ON THE UPPER MAIN SEQUENCE OF THE OPEN CLUSTER NGC 752
}



ABSTRACT. Spectra of resolution $\lambda / \Delta \lambda \sim 2 \times 10^{4}$ and good $S: N$ ratio are presented in the range containing the $7 \mathrm{Li}$ doublet at $6707 \AA$ for 9 main sequence or slightly evolved stars in NGC 752 (age $\sim 2 \times 10^{9}$ years). We investigate the suggested main sequence bimodality using spectroscopic indications of binarity and high rotational velocity, as well as the $\mathrm{Li}$ abundance to supplement previous photometry.

\section{INTRODUCTION}

NGC 752 is a moderately old open cluster, with age estimated by Demarque (1980) as $1.7 \times 10^{9}$ years. Several photometric studies most recently Twarog (1983), have shown an apparently double main sequence. $7 \mathrm{Li}$ is a probe of time-dependent processes; its abundance is one tool to explore cluster evolution. Hobbs and Pilachowski (1986, HP) have published main sequence Li abundances showing warmer stars with values up to $\log N(L i)=3.2$, a "gap" at mid-F (cf. Boesgaard and Tripicco, 1986 for the Hyades), and a fall-off from late $F$ through G. All the stars in HP lie on the "blue" main sequence, showin in Fig. 1. On this HR diagram using data from Eggen (1963) we also show the 9 stars in the present study.

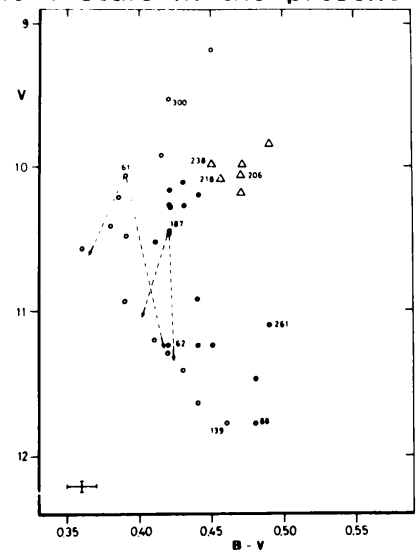

Fig. 1: HR diagram for NGC 752.

Key:

- Blue MS.

- Red MS

$\Delta$ Slightly evolved group Suggested decomposition of binaries into components

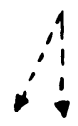

G. Cayrel de Strobel and M. Spite (eds.), The Impact of Very High S/N Spectroscopy on Stellar Physics, 473-476. (1) 1988 by the IAU. 


\section{OBSERVATIONS}

Data are from the cassegrain focus spectrograph of the $2.5 \mathrm{~m}$ Isaac Newton Telescope (La Palma; 28-30 July 1986 and 16/17 October 1986). Sltt limited spectral resolution was $\sim 0.4 \AA, 2$ pixels of the $70 \%$ q efficient CCD camera at $\lambda 6707 \AA$. Standard reduction included flat-fielding, calibration via emission lamp, and cosmic ray spike removal. For stars in range $m_{v}=10-12, s: N$ for exposures of 30-60 minutes was 100-150; the brightest object gave $S: N=200$. Abundances were from curves of growth derived from Kurucz (1979) models, carefully abstracting the $\mathrm{Fe}$ line at $6707.44 \mathrm{~A}$, with $\log g=4.0$, microturbulence $1.5 \mathrm{~km} \mathrm{~s}^{-1}$, and Teff from literature photometry (eg. Eggen, 1963; Twarog, 1983). For more detail see Rebolo and Beckman (1987).

\section{POSSIBLE CAUSES OF BIMODALITY}

(a) Binarity. A binary will, in general, appear displaced upwards and redwards in the HR diagram. If the two stars are of equal mass, the displacement is 0.75 mag brighter. Pilachowski et al. (1986) measured the parameters of the known spectroscopic binary $H 300$ which is on the upper red MS (see Fig. 1).

(b) Rotation. It is known (eg. Collins and Sonneborn, 1977) that rotation reddens a star's flux, and could therefore contribute to produce a shifted MS.

(c) Age. It is possible that the red MS is older than the blue. The HR diagram separation would correspond to an age difference of $\sim 3 \times 108$ years (Ciardullo and Demarque, 1979) not excluded by the overall cluster age. A test could be to observe $\mathrm{Li}$ later than G2, using depletion as a chronometer but for stars with $m_{v}>12.5$ this is not easy. For hotter stars a bimodal metallicity could indicate an age split.

\section{OUR Li RESULTS}

These are summarized in Table 1.

Table 1.

\begin{tabular}{|c|c|c|c|c|c|c|c|c|c|}
\hline $\begin{array}{l}\text { Star } \\
\text { (1) }\end{array}$ & $B-V$ & V & $\begin{array}{l}\text { Teff } \\
(2,3)\end{array}$ & $\begin{array}{c}W(m \AA) \\
(4)\end{array}$ & $\log N(L i)$ & MS & $\begin{array}{l}\text { Bin } \\
(5)\end{array}$ & $\begin{array}{l}\text { Rot } \\
\text { (7) }\end{array}$ & $\begin{array}{c}\text { "gap" } \\
(10)\end{array}$ \\
\hline $\mathrm{H} 61$ & 0.39 & 10.06 & 6810 & 54 & 3.05 & B & Yes ${ }^{6}$ & & -- \\
\hline H62 & 0.42 & 11.24 & 6740 & 63 & 3.1 & B & - & $-\cdots$ & -- \\
\hline H88 & 0.48 & 11.78 & 6240 & $<25$ & $<2.2$ & $\mathrm{R}$ & -- & Yes ${ }^{*}$ & Yes \\
\hline H139 & 0.46 & 11.79 & 6420 & $<40$ & $<2.7$ & B & --- & -- & Yes \\
\hline $\mathrm{H} 187$ & 0.42 & 10.45 & 6660 & $31 / 18$ (9) & $3.1 / 2.8$ & $\mathrm{R}$ & Yes & -- & -- \\
\hline H206 & 0.47 & 10.06 & 6440 & 74 & 3.05 (8) & $N$ & -- & Yes & --- \\
\hline $\mathrm{H} 218$ & 0.455 & 10.09 & 6510 & 84 & 3.1 (8) & $\mathrm{N}$ & -- & Yes & --- \\
\hline H238 & 0.45 & 9.98 & 6360 & 82 & 3.0 (8) & $N$ & -- & Yes & --- \\
\hline $\mathrm{H} 261$ & 0.49 & 11.15 & 6410 & 42 & 2.6 & $R$ & -- & -- & -- \\
\hline
\end{tabular}


(1) Heinemann's (1926) identification.

(2) (3) Hearnshaw's (1974) calibration using $H \beta$ photometry of Criawford and Barnes (1970).

(4) Fe I line at 6707.441 A included. B "blue" MS; R "red" MS; N neither MS.

(5) Spectroscopic indication of binarity.

(6) Component estimates: $T_{\text {eff }}=7000 \mathrm{~K}, \log \mathrm{N}(\mathrm{Li})=3.3 ; \mathrm{T}_{\text {eff }}=6700 \mathrm{~K}$, $\log N(\mathrm{Li})=2.7$.

(7) Position in Fig. 1 possibly affected by rotation.

(8) Sub-giants with little Li depletion.

* Low $v \sin i$, but rotational reddening needed to explain Li "gap" position.

(9) Two component estimates. $T_{\text {eff }}=6900 \mathrm{~K}, \log \mathrm{N}(\mathrm{Li})=3.1 ;$ Teff $=6700$ $K, \log N(L i)=2.8$.

(10) $\mathrm{Li}$ abundance possibly reduced as star falls in the "F gap" for $\mathrm{Li}$

(Boesgaand and Trippico, 1986).

The short format precludes details of our physical inferences, which

will be presented elsewhere (Rebolo and Beckman, 1987).

\section{CONCLUSIONS}

(a) The initial undepleted $L i$ abundance in NGC 752 was $\log N(L i) \simeq 3.1$ (cf. HP, 1986).

(b) A group of somewhat evolved stars shows no Li depletion.

(c) We identify two new spectroscopic binaries (H61, H187).

(d) We identify two clear rapid rotators (H206, H218).

The presence of two near parallel MS branches in NGC 752 appears to be something of artefact due to the small sample (some 35) of stars which make up the cluster. Future studies with a greater fraction of the cluster stars would help to decide this issue.
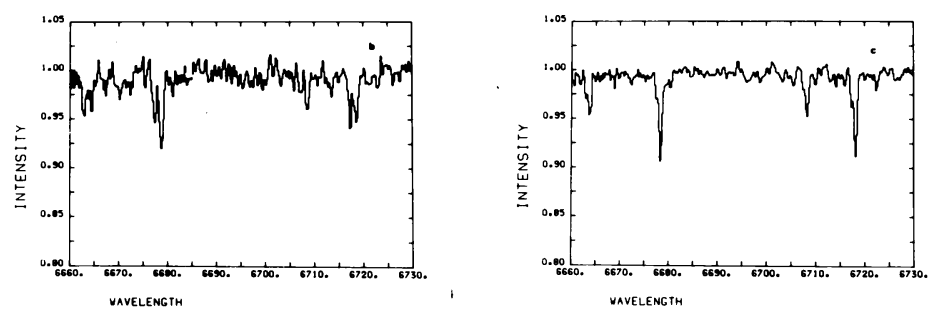

Fig. 2: b Spectrum of H187 showing clear evidence of binarity in line splittings. c Spectrum of $\mathrm{H} 61$ showing evidence for binarity with weak secondary components to the lines.

\section{References}

Boesgaard, A.M., Tripicco, M.J.: 1986, Astrophys. J. 302, L49.

Ciardullo, R.D., Demarque, P.: 1979, Dadley Obs. Rep. 14, 317.

Collins, G.W., Sonneborn, G.H.: 1977, Astrophys. J. Suppl. Ser. $34,41$. Crawford, D.L., Barnes, J.V.: 1970, Astron. J. 75, 946.

Demarque, P.: 1980, IAU Symp. No. 85, "Star Clusters" Ed. J.E. Hesser (Dordrecht, Reidel), 281. 
Eggen, O.J.: 1963, Astrophys. J. 138, 356.

Heinemann, R.: 1926, Astr. Nach. 227, 213.

Hearnshaw, J.B.: 1974, Astron. Astrophys. 34, 263.

Hobbs, L.M., Pilachowski, C.: 1986, Astrophys. J. 309, L17.

Michaud, G.: 1986, Astrophys. J. 302, 650.

Pilachowski, C., Willmarth, D.W., Halbedel, E., Mathieu, R.D., Hobbs, L.M., Milkey, R.W., Saha, A.: 1986, PASP 98, 1321.

Rebolo, R., Beckman, J.: 1987, (In preparation).

Twarog, B.A.: 1983, Astrophys. J. 267, 207. 